\title{
A rapidly evolving quasar population at the epoch of reionzation
}

\author{
Xiaohui Fan
}

University of Arizona, USA

\begin{abstract}
I will present results from our on-going large area survey of high-redshift quasars, which has discovered more than 20 new quasars at $z>6.5$, at the epoch of reionization, forming the first large statistical sample of EoR quasars. I will discuss the rapid evolution of quasar density at that epoch, which suggests that we are witnessing the emergence of the first supermassive black hole population. I will also present multiwavelength followup observation results, especially from ALMA and Chandra, which reveals a diverse environment of quasar activities and yields new insights into the supermassive black hole/massive galaxy co-evolution.
\end{abstract}

